IGCS20_1192

\section{COMBINED HYPERTHERMIC INTRAPERITONEAL CHEMOTHERAPY AND HYPERTHERMIC INTRATHORACIC CHEMOTHERAPY IN STAGE IVA OVARIAN CARCINOMA: A TERTIARY CARE CENTRE EXPERIENCE}

N Kumar*, MD Ray. AllMS, India

\subsection{6/ijgc-2020-IGCS. 169}

Introduction Multidisciplinary team approach with hyperthermic intrathoracic chemotherapy (HITHOC) is a reasonable treatment option for ovarian carcinoma with malignant pleural effusion or pleural deposits. Performing cytoreduction surgery (CRS) with hyperthermic intraperitoneal chemotherapy (HIPEC) and HITHOC is a technically demanding procedure.

Methods The data has been collected from a prospectively maintained computerized database. We are sharing our experience of 3 patients with stage IVA ovarian carcinoma patients, which were treated with CRS with HIPEC/HITHOC.

Results Three patients were age 29, 45 and 46 years. All patients were operated after neoadjuvant chemotherapy. Mean cytoreduction surgery and HIPEC/HITHOC time were 370 and 55 minutes respectively. Peritoneal carcinoma indices were 23, 2 and 15 respectively. Cisplatin drug was used in 2 patients with $100 \mathrm{mg}$ dosage at $42^{\circ} \mathrm{C}$ temperature. Mitomycin $(30 \mathrm{mg}$ ) was used in one patient. Normal saline was used as perfusate. Completeness of cytoreduction scores of 0 and 1 (CC-0/1) were achieved in 2 and 1 patients respectively. One patient developed Clavien Dindo grade III complication in the post-operative period. All patients received adjuvant Platinum-based chemotherapy because of histological residual disease. All patients are alive until the last visit with a mean follow-up period of 19 months. One patient had recurrent pleural metastasis on follow up.

Conclusions HIPEC/HITHOC is an effective, feasible and safe therapeutic option to prevent recurrence in stage IVA ovarian carcinoma. The postoperative morbidity is acceptable with no recorded mortality. Also, the quality of life improves with HITHOC in the patient having respiratory distress due to malignant pleural effusion/deposits.

\section{IGCS20_1193}

198 GERMLINE AND SOMATIC BRCA1/2 GENE MUTATIONAL STATUS AND CLINICAL OUTCOMES IN EPITHELIAL PERITONEAL, OVARIAN, AND FALLOPIAN TUBE CANCER

S Kim*, M Lee, H Kim, J Kim. Seoul National University College of Medicine, South Korea

10.1136/ijgc-2020-IGCS.170

Purpose To present a single institutional experience with BRCA1/2 gene tests and the effects of pathogenic mutations
Germline test $(\mathrm{n}=475)$

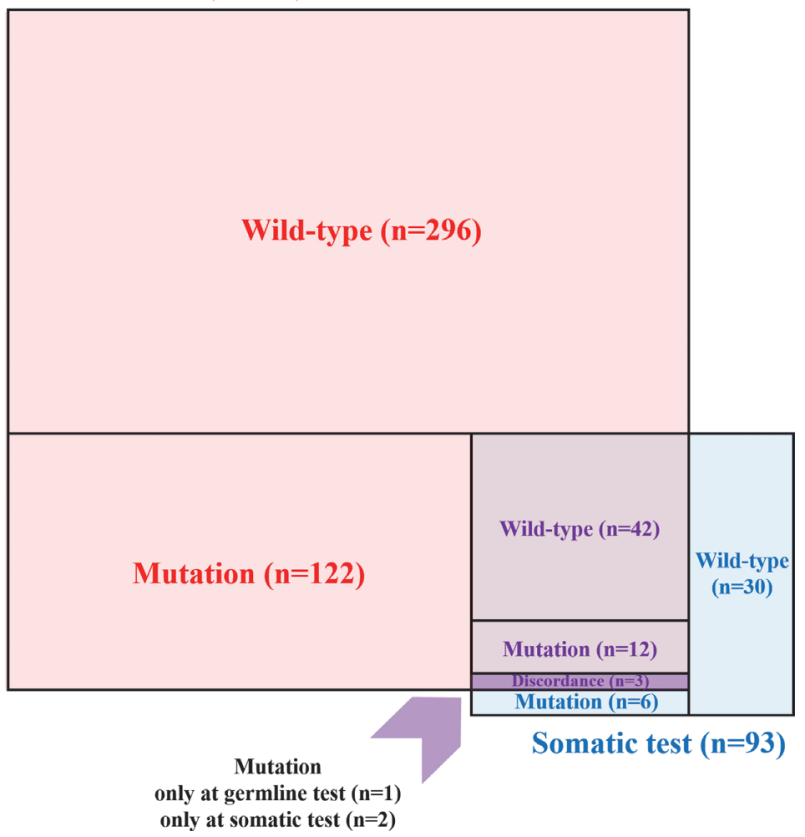

Abstract 198 Figure 1

in epithelial peritoneal, ovarian, and fallopian tube cancer (POFTC) on survival outcomes.

Methods We identified patients with epithelial POFTCs who underwent BRCA1/2 gene testing by either germline or somatic methods between March 2007 and March 2020. Based on the BRCA1/2 test results, patients were divided into BRCA mutation and wild-type groups, followed by comparisons of clinicopathologic characteristics and survival outcomes after primary treatment.

Results The annual number of POFTC patients who received BRCA1/2 gene tests increased gradually. In total, 511 patients were included and BRCA1/2 mutations were observed in $143(28.0 \%)$. Among 57 patients who received both germline and somatic tests, three (5.3\%) showed discordant results from the two tests. Overall, no differences in progression-free survival ( $\mathrm{PFS} ; \mathrm{P}=0.467$ ) and overall survival $(\mathrm{P}=0.641)$ were observed between the BRCA mutation and wild-type groups; however, multivariate analyses identified BRCA1/2 mutation as an independent favorable prognostic factor for PFS (adjusted HR, 0.765; 95\% CI, 0.5930.987; $\mathrm{P}=0.040$ ). In 389 patients with FIGO stage III-IV, different results were shown depending on primary treatment strategy: while BRCA $1 / 2$ mutation significantly improved PFS in the subgroup of neoadjuvant chemotherapy (adjusted HR, 0.619; 95\% CI, 0.385-0.995; P=0.048), it did not affect patient PFS in the subgroup of primary debulking surgery (adjusted HR, 0.759; 95\% CI, $0.530-1.089 ; \mathrm{P}=0.135$ ).

Conclusions BRCA1/2 mutations are frequently observed in patients with epithelial POFTCs, and such patients showed better PFS than did those harboring wild-type BRCA1/2. 